\title{
Towards reproducible experimental studies for non-convex polyhedral shaped particles
}

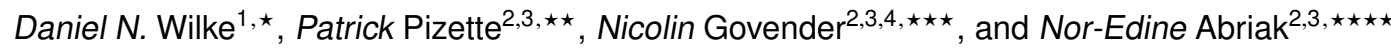 \\ ${ }^{1}$ Centre for Asset and Integrity Management, University of Pretoria, Pretoria, 0086, South Africa \\ ${ }^{2}$ Mines-Douai, LGCgE GCE, F-59508 Douai, France \\ ${ }^{3}$ Université de Lille, 59650 Lille, France \\ ${ }^{4}$ Research Center Pharmaceutical Engineering, GmbH, Graz, Austria
}

\begin{abstract}
The packing density and flat bottomed hopper discharge of non-convex polyhedral particles are investigated in a systematic experimental study. The motivation for this study is two-fold. Firstly, to establish an approach to deliver quality experimental particle packing data for non-convex polyhedral particles that can be used for characterization and validation purposes of discrete element codes. Secondly, to make the reproducibility of experimental setups as convenient and readily available as possible using affordable and accessible technology. The primary technology for this study is fused deposition modeling used to 3D print polylactic acid (PLA) particles using readily available 3D printer technology. A total of 8000 biodegradable particles were printed, 1000 white particles and 1000 black particles for each of the four particle types considered in this study. Reproducibility is one benefit of using fused deposition modeling to print particles, but an extremely important additional benefit is that specific particle properties can be explicitly controlled. As an example in this study the volume fraction of each particle can be controlled i.e. the effective particle density can be adjusted. In this study the particle volumes reduces drastically as the non-convexity is increased, however all printed white particles in this study have the same mass within $2 \%$ of each other.
\end{abstract}

\section{Introduction}

The two pillars on which science has been build is theory and experimentation [1]. Mathematical modeling initially aims to describe experimentally observed phenomena to ultimately predict the physical response of a system without requiring experimental observation, once it has been properly characterized and validated [2]. Mathematical models are often required to be characterized by conducting inverse analysis. Characterization aims to identify the essential information required to appropriately identify the respective mechanisms to be characterized in a mathematical model, although this is not always fully understood or appreciated. Having achieved this would allow for a predictive model over the domain of responses for which the respective mechanisms have been properly identified.

The availability of experimental data is critical for modelers to develop new models and also to characterize existing models. Numerous empirical studies have been conducted over the years [3], both at the lab scale and industrial scale. Experimental discrete element studies that have proven to be valuable include angle of repose investigations [4], particle shear interactions [5], hopper discharge [6], ball mill [7, 8] and dynamic impulse appli-

\footnotetext{
^e-mail: nico.wilke@up.ac.za

$\star \star$ e-mail: patrick.pizette@mines-douai.fr

$\star \star \star$ e-mail: govender.nicolin@gmail.com

$\star \star \star \star$ e-mail: nor-edine.abriak@mines-douai.fr
}

cations [9]. Numerous industrial applications have been studied in detail, but a common thread runs through all these studies, regarding the diversity of particle shapes that are considered. In these studies, particle shapes are almost exclusively convex with angularity, density, aspect ratio, moisture content etc. mainly investigated, however, nonconvexity of particles remains largely elusive in experimental studies.

In this study we consider the random packing of nonconvex polyhedral shaped particles as well as the hopper discharge of non-convex polyhedral shaped particles. However, to conduct a study in a laboratory is merely one part of the challenge. To allow for experimental studies that are reproducible when the non-convexity of particles are considered is an additional challenge but an important one for our scientific community. Hence, the goal of this study is to investigate the effect of the degree of non-convexity of particle shapes on the random packings in rectangular containers as well as the hopper discharge for flat bottomed hoppers such that it is completely reproducible by someone else, somewhere else, in the world.

Towards our first goal we consider particles that scale naturally between convex and varying degrees of nonconvex shapes. We rely on carefully selected particles that can scale between convex and non-convex shapes using compact parameterizations. 


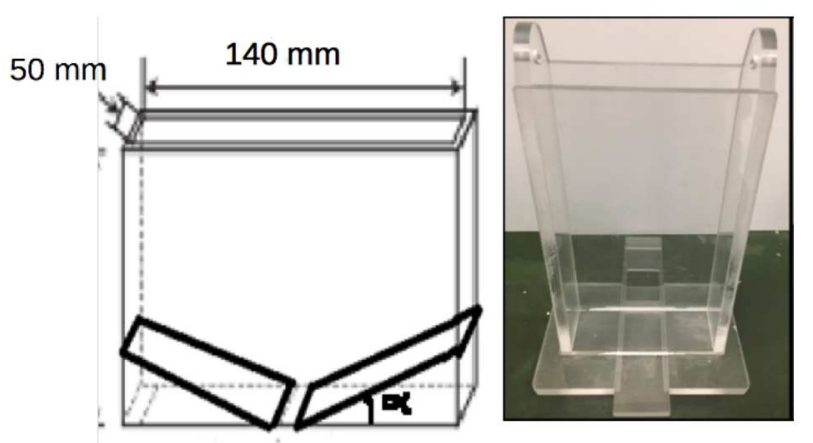

Figure 1. Schematic of the designed hoppers, with a width of $140 \mathrm{~mm}$ and a depth of $50 \mathrm{~mm}$, while the hopper opening is $50 \mathrm{~mm}$. From left to right are three manufactured hoppers, flat bottomed hopper ( $\alpha=0$ ) used in this study as well as, $\alpha=30^{\circ}$ and $\alpha=60^{\circ}$ degree hoppers.

\section{Reproducible Experiments}

The plans and materials used for the container are given in detail and limited to standard components and materials that can be sourced internationally. The raw experimental data for each experiment is made available to allow for the extraction and processing of additional data. More importantly, this allows for comparison to other researchers' experimental results. Ideally, other researchers would make their experimental results available to improve the statistical significance of the experimental data on a large number of experiments for a variety of investigations. In addition, this may inspire numerous educational opportunities to make granular flow available to wider audiences at younger ages.

The hopper considered in this study is depicted by a schematic drawing and the actual manufactured hopper from Plexiglas ${ }^{1}$ in Figure 1 . The discharge width is not indicated in the drawing but is $50 \mathrm{~mm}$. The interior of the hopper coincides with the smooth finish of the Plexiglass. The components were CNC manufactured and manually assembled at the Fabrication Laboratory (FabLab) at École des Mines de Douai, France.

\section{Convex to Non-convex Particles}

Towards our second goal of simplifying the replication of our experimental setup we base all particles on the ubiquitous fused deposition modeling (FDM) manufacturing approach [10]. All particles are 3D printed using standard white and black polylactic acid (PLA) [11]. In addition, all printed particles are made available as STereoLithography (STL) files for researchers to download and print on their own 3D printers. In addition the 3D printer settings are also made available to enhance reproducibility after

- Density: $1.18 \mathrm{~g} / \mathrm{cm}^{3}$ at the standard state of $25^{\circ} \mathrm{C}$ and $100 \mathrm{kPa}$,

- CAS Number: 9011-14-7

- IUPAC ID: Poly(methyl 2-methylpropenoate).

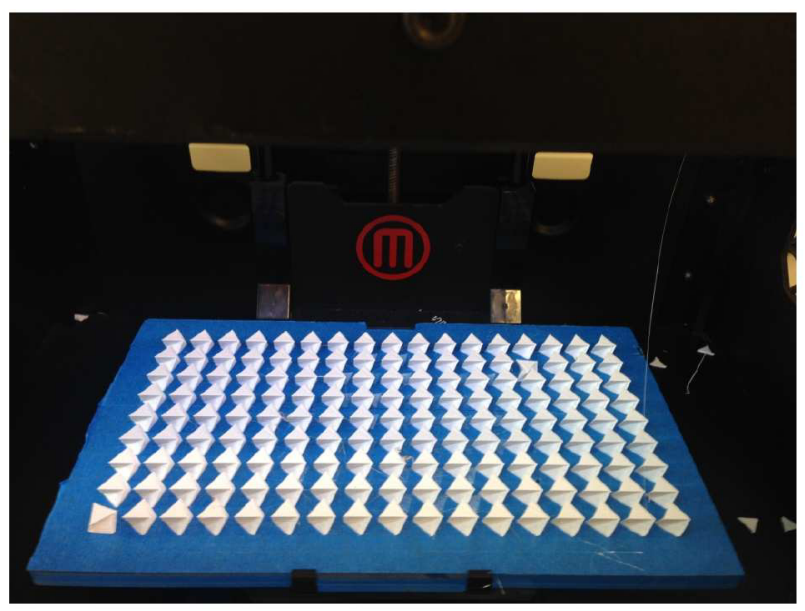

Figure 2. Makerbot Replicator utilized to print 153 (9x17) particles per batch using 100 micron layer resolution.

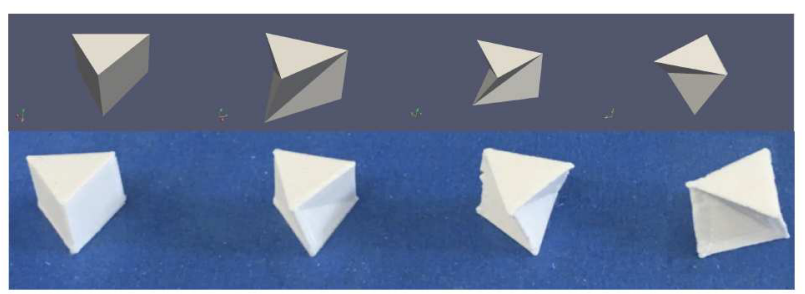

Figure 3. Four polyhedra types used in this study, starting with the triangular prism $\left(0^{\circ}\right.$ twist $)$ at the most left of the image. Three Schönhardt polyhedra are formed, from left to right, by applying $15^{\circ}, 30^{\circ}$ and $45^{\circ}$ twist respectively to the triangular prism. The top row indicates the associated rendered particles while the bottom row depicts the actual printed particles using white PLA.

a standard calibration has been performed. The particles are printed in a batch that is dependent on the size of the printer as depicted in Figure 2. The four printed particle types are depicted in Figure 3 in addition to sample STL objects of the particles.

Before we proceed, we need to establish terminology of what we mean by varying between convex and degrees of non-convex shapes. Hence, we need a way to quantify the degree of non-convexity of a particle to allow us to quantify this concept appropriately. An example of a particle that scales naturally between convex and nonconvex shapes is the Schönhardt polyhedron [12] and the variations thereof considered in this study. The Schön-

Table 1. Particle properties for the $0 \circ, 15 \circ, 30 \circ$ and $45 \circ$ twisted triangular prism and the mass of the white and black particles.

\begin{tabular}{l|llll}
\hline Particle Type & $0 \circ$ & $15 \circ$ & $30 \circ$ & $45 \circ$ \\
\hline Print VF & $20 \%$ & $28 \%$ & $36 \%$ & $100 \%$ \\
Number Shells & 1 & 1 & 1 & 1 \\
\hline Mass W kg/k (Min) & 0.3353 & 0.3352 & 0.3351 & 0.3351 \\
Mass W kg/k (Max) & 0.3354 & 0.3354 & 0.3353 & 0.3351 \\
\hline Mass B kg/k (Min) & 0.3630 & 0.3646 & 0.3648 & 0.3643 \\
Mass B kg/k (Max) & 0.3654 & 0.3652 & 0.3654 & 0.3655 \\
\hline
\end{tabular}




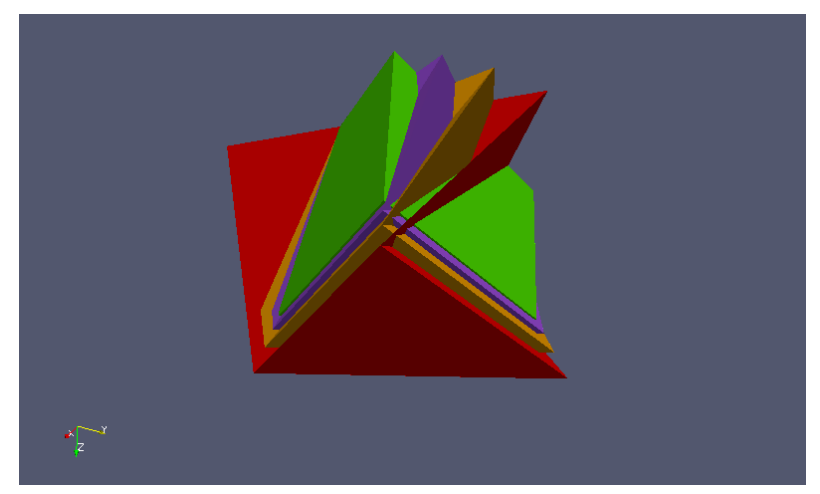

Figure 4. Schönhardt polyhedron is generated by twisting a triangular prism (green), while the edges of the triangles on opposite sides of the prism are connected to form edges that form non-convex particles. Indicated are $15^{\circ}$ (purple), $30^{\circ}$ (orange) and $45^{\circ}$ (red) twist of the one triangular base relative to the other.

hardt polyhedron is well known in geometrical topology as the simplest polyhedron that cannot be tessellated without adding an additional vertex. In this study, we propose the twist angle as a single scalar measure for the degree of non-convexity of a Schönhardt polyhedron particle. The twist angle scales between 0 (convex) and 45 (most extreme non-convex particle). Needless to say, a single scalar measure to quantify the extent of non-convexity of a single particle is inherently limited. However, when considering the convexity to non-convexity measure for the same particle that has the same size and topology, that includes texture, sub-structure and global structure, it suffice for the purposes of our study. In actual fact it works surprisingly well as a quantitative measure.

The Schönhardt polyhedron consists of six vertices that are connected to form eight surfaces that encapsulates a non-convex volume. The initial shape when no twist is applied is a convex triangular prism as depicted, by the green particle, in Figure 4. As the twist of three vertices that form a triangle is applied relative to the other triangular surface, a non-convex shape results. Hence, the twist angle is the mechanism to scale the same topological particle between a convex particle and various non-convex particles, while keeping the aspect ratio the same. In Figure 4, three twist angles of $15^{\circ}, 30^{\circ}$ and $45^{\circ}$ are depicted in purple, orange and red respectively. To ease the depiction of the particles the height is reduced, although the height of the printed particles remained the same.

The volume of the particles changes significantly as the twist angle is adjusted implying significant variations in particles mass between particle types. Fortunately, FDM allows for the density of the material to be controlled by changing the volume fraction of the printed material such that the particle mass only has a $2 \%$ variation between particle types for white and black particles respectively. This is a significant benefit of FDM in that the effective density of the particle can be changed to suit the investigation at hand. The properties for the four particle types are tabulated in table 1 , and include the print volume fraction (VF), number of shells as well as the maximum and
Table 2. Maximum height $(\mathrm{cm})$ of the random packing of the $0^{\circ}, 15^{\circ}, 30^{\circ}$ and $45^{\circ}$ twisted Schönhardt polyhedron.

\begin{tabular}{l|llll}
\hline Packing & $0 \circ$ & $15 \circ$ & $30 \circ$ & $45 \circ$ \\
\hline 1 & 12.4 & 12.2 & 12.7 & 12.9 \\
2 & 12.3 & 12.2 & 12.6 & 12.9 \\
3 & 12.5 & 12.3 & 12.7 & 13.0 \\
4 & 12.5 & 12.4 & 12.7 & 12.8 \\
5 & 12.5 & 12.3 & 12.6 & 12.9 \\
\hline Mean $(\mathrm{cm})$ & 12.4 & 12.3 & 12.7 & 12.9 \\
\hline Pack density $\left(\mathrm{g} / \mathrm{cm}^{3}\right)$ & 0.39 & 0.39 & 0.38 & 0.37 \\
\hline
\end{tabular}

minimum masses measured for the white (W) and black (B) particles for five independent measurements. Note, the mass difference of around $10 \%$ by merely changing the color of the PLA filament. Although, not done in this study the VF for the white and black particles could be independently determined to ensure that the particle mass remains the same between particle colors. All particles were printed with a layer resolution of 100 micron, although the surface roughness is expected to remain unaffected, experimental validation of this is still required. Surface metrology of the printed particles will form part of a future investigation. Quantification of the effect of volume fraction on the experimental inertial properties against idealized inertial properties in under investigation and will be included in a future study.

Documentation and standardization of the processes is being developed to allow for collaborative extension and reproduction of experimental investigations considering FDM within the DEM community. An online repository is under construction and will be communicated at the conference.

\section{Experimental Results}

A 1000 particles were randomly packed inside the hopper. The packing was repeated 10 times and the estimated height measured tabulated in table 2 using 5 samples. The effective density is computed for each random packing and it is clear that the $45 \circ$ has the densest packing while the $30 \circ$ particle has the least dense packing. In addition, the hopper discharge for the four particle types were conducted 15 times, while the mass as a function of time was tracked. The discharge rate graphs were then computed as well as the probability of stable arches forming for the various particle systems. The discharge at $0 \mathrm{~s}, 0.8 \mathrm{~s}, 1.6 \mathrm{~s}$ and $2.4 \mathrm{~s}$ are depicted in Figure 5 for the four particle types using a 1000 particles that are layered into 200 particles per layer.

\section{Conclusion}

Towards reproducible granular flow experiments we demonstrated that fused deposition modeling (FDM) is a meaningful technology, in particular when complex particle shapes are considered as with non-convex particles. Fused deposition modeling also allows for particle properties to be varied in isolation without implying an implicit 


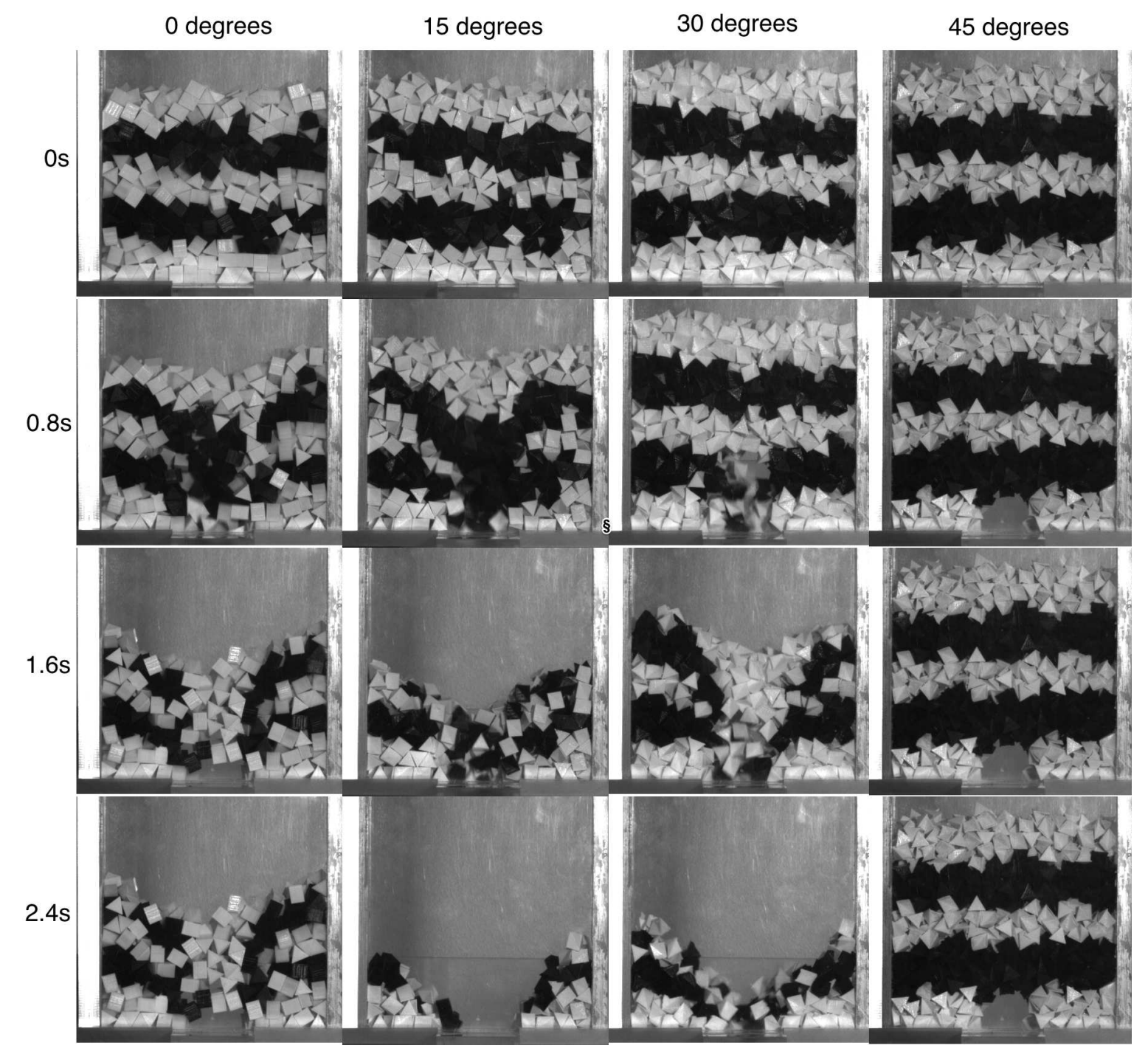

Figure 5. Flat bottomed hopper discharge for triangular prisms with $0^{\circ}, 15^{\circ}, 30^{\circ}$ and $45^{\circ}$ twist, depicted along the columns of the picture array, at $0 \mathrm{~s}, 0.8 \mathrm{~s}, 1.6 \mathrm{~s}$ and $2.4 \mathrm{~s}$, depicted along the rows of the array of pictures.

variation in other properties. As a tangible and simple example we considered the discharge of a hopper. FDM allows for constant mass particles to be manufactured even when the volume changes significantly between particle types. Therefore FDM allows for changes in particle geometry to be isolated without implying an implicit variation in other particle properties, as for example particle mass, in our study.

\section{Acknowledgments}

The due diligence of the staff at the FabLab in École des mines de Douai, France is sincerely acknowledged. Dr. Pizette and LGCgE GCE, Mines Douai for providing all experimental data documented in this paper.

\section{References}

[1] M. Vardi, Communications of the ACM 53, 5 (2010)

[2] ASME/NAFEMS pp. 1 - 4 (2006)
[3] K. Wieghardt, Annual Review of Fluid Mechanics 7, 89 (1975)

[4] Y. Li, Y. Xu, C. Thornton, Powder Technology 160, 219 (2005)

[5] C. Coetzee, Powder Technology 297, 50 (2016)

[6] N. Standish, Powder Technology 45, 43 (1985)

[7] P.W. Cleary, M.L. Sawley, Applied Mathematical Modelling 26, 89 (2002)

[8] M. Kwapinska, G. Saage, E. Tsotsas, Powder Technology 161, 69 (2006)

[9] E. Rougier, E.E. Knight, S.T. Broome, A.J. Sussman, A. Munjiza, International Journal of Rock Mechanics and Mining Sciences 70, 101 (2014)

[10] S.S. Crump, Apparatus and Method for Creating Three-Dimensional Objects (1992)

[11] B. Wittbrodt, J. Pearce, Additive Manufacturing 8, 110 (2015)

[12] E. Schönhardt, Math. Annalen 98, 309 (1928) 